\title{
Toward an understanding of nuclear substructures beyond their classical functions
}

\author{
Yuki Sakamoto $^{1} \cdot$ Takuya Sakamoto $^{2}$
}

Published online: 9 June 2020

(c) The Botanical Society of Japan 2020

The nucleus consists of several nuclear substructures, including the nuclear envelope (NE), nuclear pore complex (NPC), nuclear lamina, and nucleolus. The NE is a lipid bilayer that encloses the nucleus and physically separates it from the cytoplasm. NPCs are large protein complexes located across the NE, which function as mediators of the bidirectional exchange of macromolecules between the nucleus and cytoplasm. The nuclear lamina, lying beneath the inner nuclear membrane, is a mesh-like structure that mechanically supports the nucleus to maintain its shape. The nucleolus is a prominently dense region in the nucleus, which plays a central role in ribosomal RNA transcription and ribosome biogenesis. In the past decade, technological advances in chromatin analyses such as chromatin live imaging, super-resolution imaging, chromatin immunoprecipitation, and chromatin conformation capture have enabled more detailed insight into the spatiotemporal chromatin organization. These efforts have revealed the additional crucial roles of nuclear substructures in DNA replication, DNA damage repair, gene expression, and genome stability by regulating chromatin organization beyond their classical functions. This special issue of the JPR international symposium entitled "New aspects of functional plant nuclear architecture" provides four reviews and one original research article regarding the functions of the nuclear substructures in regulating chromatin organization in plants.

The proteomic analysis performed by Tamura et al. (2010) identified a large number of components of the Arabidopsis

Takuya Sakamoto

sakataku@rs.tus.ac.jp

1 Department of Biological Sciences, Graduate School of Science, Osaka University, 1-1 Machikaneyama-cho, Toyonaka, Osaka 560-0043, Japan

2 Department of Applied Biological Science, Faculty of Science and Technology, Tokyo University of Science, 2641 Yamazaki, Noda, Chiba 278-8510, Japan thaliana NPC, termed nucleoporins (NUPs), which help to promote understanding of plant NPC molecular functions. Tamura (2020) further reviews the cutting-edge knowledge of NPC functions in the regulation of gene expression. He focused on three different styles of NPC-mediated gene activation: (i) transport of the transcriptional machinery from the cytoplasm to the nucleus, (ii) providing a scaffold for assembly of the transcriptional machinery at the nuclear periphery, and (iii) formation and maintenance of gene loops, which allow for the rapid reactivation of genes.

In the metazoan nucleus, the nuclear lamina is mainly composed of filamentous protein lamins; however, the ortholog of lamin is not conserved in plants. Instead, in $A$. thaliana, crowded nuclei proteins (CRWNs) are considered to be the main components of the nuclear lamina (Sakamoto and Takagi 2013). In this special issue, Sakamoto (2020) provides a nice overview of plant nuclear lamina functions based on the analyses of CRWNs in A. thaliana. This review covers the intimate involvement of CRWNs in regulating chromatin organization, repression of specific genes related to immunity, and DNA damage repair. In addition, the review discusses the mechanism of gene silencing via the action of CRWNs at the nuclear periphery.

Thus far, the genomic regions associated with the nucleolar periphery, termed nucleolus-associated domains (NADs), have been identified in A. thaliana (Pontvianne et al. 2016). A. thaliana NADs are enriched in pseudogenes and transposable elements, in addition to sub-telomeric regions. The anchoring of specific genomic regions to the nucleolus periphery is considered to contribute to the spatial organization of chromatins, in which a nucleolar protein, nucleolin 1 (NUC1), plays pivotal roles (Pontvianne et al. 2016). In this special issue, Picart-Picolo et al. (2020) report the findings from an investigation of the effects of heat stress on the composition of NADs, revealing that the NADs composition remained stable during heat stress. However, the nucleolar structure was altered under heat stress, which slowed down 
the rate of ribosome biogenesis. These findings suggest that the function of the nucleoli in spatial chromatin organization is independent of another major function of the nucleolus in ribosome biogenesis.

Oko et al. (2020) review the spatial positioning of organized heterochromatin such as centromeres and telomeres in nuclear space, with a primary focus on studies conducted with $A$. thaliana. They comprehensively cover the nuclear factors involved in the positional regulation of centromeres and telomeres and discuss the biological importance of these regulations. They highlight the critical roles of the nucleoplasmic protein complex condensin II, the nuclear peripheral proteins including NE proteins, and the nuclear lamina CRWN proteins in proper centromere positioning in A. thaliana. They further claim that proper centromere positioning has little effect on gene expression control, despite its impact on the regulation of genome-wide chromatin organization, including centromeric heterochromatin in A. thaliana. Furthermore, Oko et al. (2020) provide evidence for the involvement of the nucleolar protein NUC1 in telomere positioning and discuss its significance in the maintenance of telomere organization in A. thaliana.

The application of genome-wide derivatives of a chromosome conformation capture method, Hi-C, has enabled mapping of the three-dimensional (3D) chromatin organization on a genome-wide scale in plants (Feng et al. 2014; Grob et al. 2014). In this special issue, Pontvianne and Grob (2020) provide an overview of the state-of-the-art knowledge on 3D chromatin organization, mainly based on studies in A. thaliana. They explain the features of 3D chromatin organization at different scales, such as chromatin loops, topologically associated domains, the chromatin compartment, and chromosome territories, and discuss the similarities and differences between plants and animals as well as between $A$. thaliana and other plant species. In addition, they describe a novel gene-silencing system of transgenes discovered in $A$. thaliana, which is determined by the specific 3D positioning of these loci (Grob and Grossniklaus 2019). Furthermore, they discuss the significance of associations between specific chromatin regions and the nuclear lamina and nucleolus.

As described above and highlighted in this special issue, our knowledge of the functions of plant nuclear substructures in chromatin organization has rapidly increased. However, we must note that most of the knowledge gained to date has been based on analyses of A. thaliana. The features of genome organization of A. thaliana differ from those of many other plant species in terms of gene density, heterochromatin distribution, and other key characteristics. In particular, the telomere positioning of $A$. thaliana is unique among plants. Considering these facts, further chromatin analyses should be attempted by mutating nuclear substructures in other plant species as well as digging deeper into the molecular actions of nuclear substructures in regulating chromatin organization in A. thaliana.

\section{References}

Feng S, Cokus SJ, Schubert V, Zhai J, Pellegrini M, Jacobsen SE (2014) Genome-wide Hi-C analyses in wild-type and mutants reveal high-resolution chromatin interactions in Arabidopsis. Mol Cell 55:694-707

Grob S, Grossniklaus U (2019) Invasive DNA elements modify the nuclear architecture of their insertion site by KNOT-linked silencing in Arabidopsis thaliana. Genome Biol 20:120

Grob S, Schmid MW, Grossniklaus U (2014) Hi-C analysis in Arabidopsis identifies the KNOT, a structure with similarities to the flamenco locus of Drosophila. Mol Cell 55:678-693

Oko Y, Ito N, Sakamoto T (2020) The mechanisms and significance of the positional control of centromeres and telomeres in plants. $\mathrm{J}$ Plant Res. https://doi.org/10.1007/s10265-020-01202-2

Picart-Picolo A, Picart C, Picault N, Pontvianne F (2020) Nucleolusassociated chromatin domains are maintained under heat stress, despite nucleolar reorganization in Arabidopsis thaliana. J Plant Res. https://doi.org/10.1007/s10265-020-01201-3

Pontvianne F, Grob S (2020) Three-dimensional nuclear organization in Arabidopsis thaliana. J Plant Res. https://doi.org/10.1007/ s10265-020-01185-0

Pontvianne F, Carpentier MC, Durut N, Pavlištová V, Jaške K, Schořová Š, Parrinello H, Rohmer M, Pikaard CS, Fojtová M et al (2016) Identification of nucleolus-associated chromatin domains reveals a role for the nucleolus in 3D organization of the $A$. thaliana genome. Cell Rep 16:1574-1587

Sakamoto Y (2020) Nuclear lamina CRWN proteins regulate chromatin organization, gene expression, and nuclear body formation in plants. J Plant Res. https://doi.org/10.1007/s10265-020-01184-1

Sakamoto Y, Takagi S (2013) LITTLE NUCLEI 1 and 4 regulate nuclear morphology in Arabidopsis thaliana. Plant Cell Physiol 54:622-633

Tamura K (2020) Nuclear pore complex-mediated gene expression in Arabidopsis thaliana. J Plant Res. https://doi.org/10.1007/s1026 5-020-01177-0

Tamura K, Fukao Y, Iwamoto M, Haraguchi T, Hara-Nishimura I (2010) Identification and characterization of nuclear pore complex components in Arabidopsis thaliana. Plant Cell 22:4084-4097

Publisher's Note Springer Nature remains neutral with regard to jurisdictional claims in published maps and institutional affiliations. 